Original Research

\title{
A Case Control Study on Physical Activity and Body Mass Index Associated with Hemorrhoids
}

\section{Jalina Karim, Alif Asyraf Arispa Abdullah, A'syaratun Kamilah Zolkifle, Nur Shafizah Roslan, Suganthi Kumar and Kelvin C Shiong}

Faculty of Medicine, Universiti Kebangsaan Malaysia

\begin{abstract}
Introduction: Body mass index (BMI) and physical activity are the controversial risk factors that cause hemorrhoids. This study was conducted to determine the association between body mass index and physical activity.

Methods: This was a case-control study which involved two hundred and two patients using convenience sampling. They had to answer a questionnaire consisting of the International Physical Activity Questionnaire (IPAQ) which monitored their physical activity and their BMI was also measured. For the descriptive analysis, Chi square and an odd ratio were carried out.

Results: There was a significant association between age and hemorrhoids $(\mathrm{p}=0.02)$, in which the patients who were younger than 50 years old were more likely to have hemorrhoids compared to those who were older than 50 years old (OR=2.268, 95\%CI: 1.107-4.630). For the risk estimation calculation, the Chinese individuals were found to have a higher risk compared to non-Chinese individuals (OR=2.056, 95\% CI: 1.174-3.601). BMI was proven to be significantly associated with hemorrhoids $(p=0.043)$ Physical activities were found to not be statistically significant $(p=0.209)$. Those with a low and moderate physical activity level were 1.24 times more likely to have hemorrhoids compared to those with a high level of physical activity (OR=1.243, 95\%CI: 0.697-2.217). The confidence interval was between 0.697 and 2.217 , therefore it was not statistically significant.

Conclusion: Physical activity was not associated with the hemorrhoids. However, it was shown that good physical activity could help to regulate bowel function and therefore, the occurrence of hemorrhoids would be less likely. BMI was significantly associated with hemorrhoids.
\end{abstract}

Cite this as: Karim, J., Abdullah, A. A. A., Zolkifle, A. K., Roslan, N. S., Kumar, S., \& Shiong, K. C. (2019). A Case Control Study on Physical Activity and Body Mass Index Associated with Hemorrhoids.Jurnal Ners, 14(3si), 121-125. doi:http://dx.doi.org/10.20473/in.v14i3(si).17045

\section{INTRODUCTION}

Hemorrhoids are defined as enlarged congested patches of mucosa and submucosa of the anal cushions which can further be divided into external and internal hemorrhoids. Hemorrhoids are a very common benign anorectal disease (Lohsiriwat, 2015) and a common anorectal problem (Jay et al., 2019). This condition can be an acute or chronic in which can be categorized into four degrees on the basis of the history given by the patient (Ratto et al., 2011).

Hemorrhoids are a common condition, especially in Western countries. About half of the population

\section{ARTICLE HISTORY}

Received: Dec 26, 2019

Accepted: Dec 31, 2019

\section{KEYWORDS}

physical activity; body mass index; haemorrhoids

\section{CONTACT}

Jalina Karim

凶jalina@ppukm.ukm.edu.my

$\equiv$ Faculty of Medicine, Universiti Kebangsaan Malaysia over 40 years of age suffer from the disease, from mild through to a more severe form (Dimmer, Martin, Reeves, \& Sullivan, 1996). A statistical value for Malaysia and hemorrhoids was not found. However, a previous study showed the risk factors which consisted of obesity, irregular bowel habits (either constipation or diarrhea), s lack of exercise, a low fiber diet, increase intra-abdominal pressure due to prolonged straining, pregnancy, position during defecation, prolonged sitting or standing, and aging (Zeinab, El-Sayed, \& Taha, 2011). The current situation in Malaysia is that there is an increase of 
people who are overweight and obese among the population because of the lifestyle of having a low level of physical activity and their eating preferences (Lee, Kim, Kang, Shin, \& Song, 2014). Most of the hemorrhoid cases preferred to be self-medicated rather than seeking hospital treatment or management (Lohsiriwat, 2012). Thus, the prevalence of hemorrhoids remains unknown (Jacobs, 2018).

Even though the mechanism of obesity causing hemorrhoids is controversial, there have been several hypotheses formulated. First, it is believed to cause an increase in the intra-abdominal pressure due to the high body weight and visceral fats in which are thought to provoke the venous congestion of the distal rectum. Obesity will induce the release of inflammatory cytokines and acute phase proteins which will eventually activate the innate immune system and affect metabolic homeostasis, which contributes to the formation of hemorrhoids (Lee et al., 2014). It was proven by two studies conducted in Australia and Korea that there was an association between obesity and hemorrhoid (Lee et al., 2014; Riss et al., 2012). However, there was a study in which contradict showed that, there was no association of obesity and hemorrhoid (Peery et al., 2015).

Normally, people classify a person with a high BMI as having a low physical activity level. Nonetheless, people who are overweight or obese with an appropriate BMI will always encounter cardiovascular or metabolic disease where the main culprit is caused by low physical activity (Gang et al., 2005). A previous study proved that a sedentary lifestyle or person with a low physical activity level was found to be aligned with a low risk of developing hemorrhoids (Jacobs, 2018), whereas several studies showed that there was no association between physical activity and the risk of getting hemorrhoids (Lee et al., 2014). In relation to that, the current study is interested in finding out if there is a relation between BMI and physical activity and its effect on hemorrhoids to determine the association between the level of physical activity with the occurrence rate of hemorrhoids.

\section{MATERIALS AND METHODS}

This was a case-control study design with a convenience sampling technique approach. The study setting was at the Surgery Clinic Universiti Kebangsaan Malaysia Medical Centre (UKMMC). This study used the sample size calculation formula of case control to calculate the estimated sample size. The sample required was two hundred and two patients for both groups; hemorrhoids and non-hemorrhoids. They were invited and consented to answering a questionnaire regarding their physical activity using a standardized International Physical Activity Questionnaire (IPAQ) (Booth, 2000). Their BMI was measured and classified based on the Malaysian Clinical Practice Guideline on the Management of Obesity (Lim et al., 2000). Ethical approval was acquired from the ethical committee board UKMMC (UKM PPI/111/8/JEP-2016-172).

Body Mass Index was calculated using the following formula: weight in kilograms divided by height in meters squared. The results were classified based on the Malaysian Clinical Practice Guidelines (CPG) on the Management of Obesity (Lim et al., 2000). The weight of the participants was measured using a weighing machine where the participants were required to remove their outer garments (jacket, coat, etc), shoes and any heavy things on their body (wallet, phone, etc). For the measurement of height, the stadiometer in the clinic was used. The respondents were given 15 minutes to complete the questionnaires. The results were classified under 3 categories; low, moderate and high. Low (category 1) was for the individuals who did not meet the criteria for categories 2 or 3 , who were thus considered to be inactive. The moderate (category 2) was for any one of the following 3 criteria, which was 3 or more days of vigorous activity for at least 20 minutes per day, 5 or more days of moderate-intensity activity or walking for at least 30 minutes per day or 5 or more days of any combination of walking. High (category 3 ) was for any one of the following two criteria, which was a vigorous-intensity activity on at least three days or more days in any combination of walking, moderate-intensity or vigorous intensity activities.

The statistical analysis was conducted using IBM SPSS software version 20.0 (SPSS Inc., Chicago, IL). This study categorized the value of interest into the categorical variables which included gender, ethnicity, body mass index and physical activity, whereas age was the continuous variable. Chi Square was used to analyze the categorical variables. The mean or median was determined from the interquartile range for both parametric and nonparametric variables. An independent student t-test was used to test for differences in the analyzed parametric parameters between the groups. The Odd Ratio was used to allow for a comparison of the hemorrhoid patients relative to the control/placebo of non-hemorrhoid patients. A p-value of less than 0.05 for the two-tailed test was to be considered significant. Bar charts were used for each variable of interest to further explore them and to have a clearer view of the results.

\section{RESULTS}

Upon the questionnaire being given, the study had a total number of respondents consisting of (male $=108$, female=94) between 101 patients with hemorrhoids and 101 patients without hemorrhoids. The mean age of the respondents was $57.52 \pm 15.15$ years for hemorrhoids and $62.33 \pm 13.86$ years for those without hemorrhoids. There was a significant association between age and hemorrhoids $(p=0.02)$, whereby the patients who were younger 50 years old were more likely to have hemorrhoids compared to the patients who were older than 50 years old (OR=2.268, 95\%CI: 1.107-4.630). The Pearson Chi- 
Table 1. Background of Hemorrhoids and without Hemorrhoids

\begin{tabular}{|c|c|c|c|c|c|c|}
\hline \multirow{2}{*}{ Characteristic } & \multicolumn{2}{|c|}{ Hemorrhoids } & \multicolumn{2}{|c|}{ Without hemorrhoids } & \multirow{2}{*}{ Total } & \multirow{2}{*}{$P$ value } \\
\hline & $\mathbf{n}$ & 5 & $\mathbf{n}$ & $\%$ & & \\
\hline \multicolumn{7}{|l|}{ Gender } \\
\hline Male & 52 & 48.1 & 56 & 51.9 & 108 & 0.573 \\
\hline Female & 49 & 52.1 & 45 & 47.9 & 94 & \\
\hline \multicolumn{7}{|l|}{ Age } \\
\hline Low Age $(<50$ years old $)$ & 27 & 65.9 & 14 & 34.1 & 41 & 0.023 \\
\hline High Age ( $\geq 50$ years old) & 74 & 46.0 & 87 & 54.0 & 161 & \\
\hline \multicolumn{7}{|l|}{ Ethnicity } \\
\hline Malay & 37 & 40.2 & 55 & 59.8 & 92 & \\
\hline Chinese & 59 & 59.0 & 41 & 41.0 & 100 & $0.049 *$ \\
\hline Indian & 4 & 44.4 & 5 & 55.6 & 9 & \\
\hline Others & 1 & 100.0 & 0 & 0 & 1 & \\
\hline Chinese & 59 & 59.0 & 41 & 41.0 & 100 & 0.011 \\
\hline Non-Chinese & 42 & 41.2 & 60 & 58.8 & 102 & \\
\hline \multicolumn{7}{|l|}{ Body Mass Index (BMI) } \\
\hline Underweight & 3 & 21.4 & 11 & 78.6 & 14 & \\
\hline Normal & 32 & 48.5 & 34 & 51.5 & 66 & 0.043 \\
\hline Overweight & 45 & 60.0 & 30 & 40.0 & 75 & \\
\hline Obese & 21 & 44.7 & 26 & 55.3 & 47 & \\
\hline Obese & 66 & 54.1 & 56 & 45.9 & 122 & 0.15 \\
\hline Non-obese & 35 & 43.8 & 45 & 56.3 & 80 & \\
\hline \multicolumn{7}{|l|}{ Physical Activity Class } \\
\hline Low & 25 & 62.5 & 15 & 37.5 & 40 & \\
\hline Moderate & 43 & 47.3 & 48 & 52.7 & 91 & 0.209 \\
\hline High & 33 & 46.5 & 38 & 53.5 & 71 & \\
\hline
\end{tabular}

$0.049^{*}=$ significant association, but $50 \%$ of cells have an expected count less than 5 , therefore the result was not taken as Chi square cannot be used.

Square Test was used (Table 1 \& Table 2). For ethnicity, the numbers of respondents according to race was tabulated in Figure 1. Following the risk estimation calculation, those of Chinese ethnicity were found to have a higher risk compared to nonChinese (OR=2.056, 95\%CI: 1.174-3.601) (Table 2).

There was a significant association between BMI and hemorrhoids $(\mathrm{p}<0.05)$. Underweight individuals were less likely to have hemorrhoids compared to non-underweight people (OR=0.250 95\%CI 0.068-0.927) (Table 2). Being of a non-underweight status was therefore concluded as the total of the normal weight, overweight and obese respondents.

Physical activity was found to not be statistically significant $(p=0.209)$ (Table 1$)$. Since it was $p>0.05$, there was no significant association between the level of physical activity and hemorrhoids. Those with low and moderate physical activity (Class1) were 1.24 times more likely to have hemorrhoids compared to those with a high level of physical activity (Class 2) (OR=1.243 95\%CI 0.697-2.217). The confidence interval was between 0.697 and 2.217 , therefore it was not statistically significant compared to those with low activity and those with a moderate level of physical activity (Class 1) were more likely to have hemorrhoids (Table 2).

There was also found to be no significant association between gender and hemorrhoids $(p=0.573)$ (Table 1). This study looked for a correlation between BMI and physical activity, and there was an inverse correlation found between BMI and physical activity, but it was not significant at $\mathrm{p}=0.57$ (Table 3).

\section{DISCUSSION}

In this study, it was found that BMI was significantly associated with hemorrhoids. However, obesity was not proven to be an associated risk factor for hemorrhoids. Similar to this study, (Peery et al., 2015) showed there to be no association between obesity and the presence of hemorrhoid. However, this was in contrast with the postulated theory that obesity was associated with hemorrhoids (Lee et al., 2014; Riss et al., 2012). Obese people will have high intraabdominal pressure, contributed by their high level of body fat and visceral fat, therefore provoking venous congestion of the distal rectum and contributing to the development of hemorrhoid (Lohsiriwat, 2012). Obesity will induce the release of inflammatory cytokines and acute phase proteins, which eventually will activate the innate immune system and affect metabolic homeostasis, which contributes to the formation of hemorrhoids (Lee et al., 2014).

Nevertheless, the current study found that being underweight was less likely to predispose the individual to hemorrhoids whereas being overweight had the highest distribution among hemorrhoid patients. According to (Carter, Gabel, Zbar, Segev, \& Kopylov, 2013), there was a small but significant inverse correlation between BMI and hemorrhoids. Therefore, even though obesity was not strongly associated with hemorrhoid, it was recommended that patients with hemorrhoids should control their BMI in order to prevent the recurrence of hemorrhoids in the future. 
Table 2. Odd Ratio between Hemorrhoids and without Hemorrhoids

\begin{tabular}{lll}
\hline Characteristics & Odd ratio/0R (95\% CI) & OR Significance \\
\hline Gender (Female/ male) & $1.172(0.674-2.041)$ & Not significant \\
Age (less than 50/ more than 50) & $2.268(1.107-4.630)$ & Significant \\
Ethnicity (Chinese/ Non-Chinese) & $2.056(1.174-3.601)$ & Significant \\
BMI (Obese/Non obese & $1.515(0.859-2.674)$ & Not significant \\
BMI (Underweight/ Non-underweight & $0.250(0.068-0.927)$ & Significant \\
Physical activity (Class 1/Class 2) & $1.243(0.697-2.217)$ & Not significant \\
\hline
\end{tabular}

Table 3. Odd Ratio between Hemorrhoids and without Hemorrhoids

\begin{tabular}{lllll}
\hline Dependent variable & Independent variable & $\mathbf{r}^{2}$ & P-value in final equation & B value \\
\hline BMI & Physical Activity & 0.003 & 0.57 & -0.057 \\
\hline
\end{tabular}

In terms of age group, the current study indicated that age had a significant association with hemorrhoids. Among the patients who were younger than 50 years old, the hemorrhoid patients consisted of $65.9 \%$ compared to those without hemorrhoids which was only $34.1 \%$. Being related, patients who were younger than 50 years old were more likely to have hemorrhoids compared to those who were 50 years old and older. Clinically, hemorrhoids occur due to high anal canal pressure and it remains higher in the younger age group. This is because they have tight internal sphincter (high tone) compared to the older age group (Carter et al., 2013). This study also found that those who were Chinese had a higher risk of hemorrhoids compared with other races in Malaysia, including Malay, Indians and other races.

Surprisingly, this study found that physical activity was not associated with the risk of hemorrhoids. Similarly, a study by (Lee et al., 2014) stated that there was no significant association of the condition. A sedentary lifestyle was also associated with a reduced risk of hemorrhoid (Peery et al., 2015). However, prolonged sitting was postulated to increase the pressure on the anus, therefore resulting in hemorrhoids. It was also shown that good physical activity could help to regulate bowel function (Zeinab et al., 2011) and therefore, the occurrence of hemorrhoids would be less likely. Moreover, there were many other risk factors of hemorrhoid that were not studied in this research such as diet. A diet of high fiber is thought to reduce the risk of constipation, thus improving bowel function (Peery et al., 2015).

There were no discrepancies in terms of the presence of hemorrhoid between males and females. This study found that both genders were almost equally affected. Among the hemorrhoid patients, males made up $51.5 \%$ whereas females made up $48.5 \%$. This was in contrast to some studies which stated that females had a higher prevalence of hemorrhoids (Lee et al., 2014; Riss et al., 2012). However, it was important that this current study asked about history of pregnancy. This is because pregnancy was a well-known risk factor to contribute for hemorrhoid and estimated $85 \%$ of them would have hemorrhoid during their second or third trimester (Peery et al., 2015) therefore, pregnant woman was excluded in this study.
In short, this study concluded that BMI and age were related to the occurrence of hemorrhoid but physical activities, and obesity showed no significance. Yet, BMI and physical activity would always be related. With good and frequent physical activity, body mass index would be lower and therefore, lower risk of hemorrhoid. Hemorrhoid are one of the most common gastrointestinal diseases throughout the world (Jacobs, 2018). All of the risk factors should be studied for a better understanding of the disease in order to reduce morbidity.

\section{CONCLUSION}

Physical activity was not associated with the hemorrhoids. However, it was shown that good physical activity could help to regulate bowel function and therefore, the occurrence of hemorrhoids would be less likely. BMI was significantly associated with hemorrhoids. Therefore, health practitioners should portray their role of educating the public to change their sedentary lifestyle and unhealthy diet in order to prevent hemorrhoids for better health. This study evaluated all patients irrespective of age and gender based on the colonoscopy results to confirm the diagnosis of hemorrhoids and non-hemorrhoids, which means that we did not miss internal hemorrhoids, which would have led to a misdiagnosis and falsified data in the study. Plus, this study did not restrict the inclusion criteria as our exclusion criteria were non- Malaysians and pregnant women only. This was to ensure that we had large sample population, increasing the confidence intervals and thus increasing the precision.

The limitation of this study was age, as most of the participants were older than 50 years old. Moreover, as the International Physical Activity Questionnaire (IPAQ) Long Form (2011) measured the level of physical activity for the last 7 days, this study was unable to determine whether the patient's hemorrhoid problem was due to their past or present level of physical activity. Since the population of this study was more focused on the Chinese community, it could influence the results of the study.

This study used convenience sampling. It could be suggested to use simple random sampling in order to avoid bias in sampling techniques for future use. In addition, a multi-centre design could be suggested for further study to get a clearer picture of the issue since 
the number of obese individuals is increasing every year.

\section{REFERENCES}

Booth, M. L. (2000). Assessment of Physical Activity: An International Perspective. Research Quarterly For Exercise and Sport, 71(2), s114-120.

Carter, D., Gabel, M. B., Zbar, A., Segev, S., \& Kopylov, U. (2013). Prevalence and Clinical Associations of Haemarrhoids at screening Colonoscopy. World Journal of Colorectal Surgery, 3(2), 1-13.

Dimmer, C., Martin, B., Reeves, N., \& Sullivan, F. (1996). Squatting for the Prevention of Haemorrhoids. Townsend Letter for Doctors and Patients, 159, 67-70.

Gang, H., Jousilahti, P., Noel, C. B., Qing, Q., Laka, T. A., \& Tuomilehto, J. (2005). Physical Activity, CardiovascularRisk, Factor and Mortality Among Finnish Adults with Diabetes. Diabetes Care, 28(4).

Jacobs, D. O. (2018). Hemorrhoids. Current Opinion in Gastroenterology, 34(1), 46-49. https://doi.org/10.1097/MOG.00000000000004 08

Jay, J. I., McKenzie, C., Justin, T. B., Sharon, L. S., Reynolds, H. L., \& Emily, S. (2019). Evaluating the Accuracy of Haemorrhoids: Comparison Among Specialties and Symptom. Diseases of The Colon And Rectum, 62(4).

Lee, J., Kim, H., Kang, J., Shin, J., \& Song, J. (2014). Factors Associated with Hemorrhoids in Korean Adults: Korean National Health and Nutrition Examination Survey. Korean Journal of Family Medicine, 35(5), 227-236.
Lim, T. O., Ding, L., Zaki, M., Suleiman, A., Fatimah, S., Siti, S., ... Maimunah, A. (2000). Distribution of Body Weight, Height and Body Mass Index in a National Sample of Malaysian Adults . 108-128.

Lohsiriwat, V. (2012). Hemorrhoids: From Basic Pathophysiology to Clinical Management. World Journal of Gastroenterology, 18(17), 2009-2017. https://doi.org/10.3748/wjg.v18.i1722009

Lohsiriwat, V. (2015). Treatment of Haemorrhoids: A Coloproctologist's View. World Journal Gastroenterol, 21, 9245-9252.

Peery, A. F., Sandler, R. S., Galanko, J. A., Bresalier, R. ., Figueiredo, J. ., Ahnen, D. J., ... Baron, J. A. (2015). Risk Factors for Hemorrhoids on Screening Colonoscopy. PloS One, 10(9). https://doi.org/10.1371/journalpone.0139100

Ratto, C., Giordano, P., Donisi, L., Parello, A., Litta, F., \& Doglietto, G. B. (2011). Transanal haemorrhoidal dearterialization ( THD ) for selected fourthdegree haemorrhoids. Tech Coloproctol, 15, 191197. https://doi.org/10.1007/s10151-011-06891

Riss, S., Weiser, F. A., Schwameis, K., Riss, T., Mittlbock, M., Steiner, G., \& Stift, A. (2012). The Prevalence of Hemorrhoids in Adults. International Journal of Colorectal Disease, 27(2), 215-220. https://doi.org/10.1007/s(1)384-011-1316-3

Zeinab, H. A., El-Sayed, N. ., \& Taha, N. (2011). Effects of Conservative Measures in Improving Hemorrhoid Stages and Relieving Symptoms Among Patients with Hemorrhoid. Journakl of American Science, 7(9), 53-65. 